\title{
PREVALÊNCIA DOS QUESTIONÁRIOS DE SAÚDE GLOBAL E SAÚDE BUCAL NA AVALIAÇÃO DA QUALIDADE DE VIDA EM PACIENTES COM CÂNCER DE CABEÇA E PESCOÇO SUBMETIDOS À RADIOTERAPIA
}

Prevalence of global health and oral health questionnaires in the assessment of quality of life in patients with head and neck cancer submitted to radiotherapy

Predominio del cuestionario de salud global y de salud oral en la evaluación de la calidad de vida en pacientes con cáncer de cabeza y cuello sujetos a radioterapia

Raquel D'Aquino Garcia Caminha ${ }^{1}$, Isabela Camera Messias Bueno ${ }^{1}$, Debora Foger Teixeira $^{1}$, Guilherme Simpione ${ }^{1}$; Paulo Sérgio da Silva Santos ${ }^{1 *}$

${ }^{1}$ Departamento Estomatologia, Patologia and Radiologia, Faculdade de Odontologia de Bauru, Universidade de São Paulo, Bauru, Brasil.

*Correspondência: Paulo Sérgio da Silva Santos, Endereço: Alameda Octávio Pinheiro Brisola, 9-75, CEP: 17012901, Bauru, São Paulo, Brasil._Tel: 55 (14) 3226-6113, Fax: 0055 (14) 3223-4679

e-mail: : paulosss@fob.usp.br

\section{Artigo recebido em 06/05/2020 aprovado em 17/06/2021 publicado em 20/10/2021.}

\section{RESUMO}

$O$ câncer de cabeça e pescoço (CCP) representa um grupo de tumores malignos sendo a radioterapia (RxT) um tratamento amplamente empregado, entretanto possui efeitos colaterais como mucosite oral, trismo, xerostomia, hipossalivação, dentre outros. Foram desenvolvidos questionários para avaliar a qualidade de vida (QV) desses pacientes e o objetivo dessa revisão integrativa foi avaliar a prevalência dos questionários para avaliação da saúde bucal e geral dos pacientes submetidos a RxT para CCP na literatura. Buscas nas bases de dados PubMed ${ }^{\circledR}$ e Lilacs foram realizadas com descritores: "Oral health", "Quality of life", "Head and neck neoplasms" e "Radiotherapy" e "Saúde bucal", "Qualidade de vida", "Neoplasias de cabeça e pescoço" e "Radioterapia"; critérios de inclusão: artigos de questionários de $Q V$ em pacientes com CCP tratados com RxT com/sem outros tratamentos antineoplásicos; tipo de estudo: randomizado, não randomizado, de viabilidade, transversal, regressão linear, prospectivo, prospectivo longitudinal e prospectivo randomizado; disponiveis online; período: 2000-2020 e publicados em português/inglês. Total de 18 artigos selecionados utilizando questionários de saúde geral e global em paciente com CCP. Conclui-se que o questionário da Organização Europeia para Pesquisa e Tratamento do Câncer (versão específica para CCP H\&35 foi o mais aplicado seguido de sua versão voltada para saúde global (C30).

Palavras-chave: Neoplasias de Cabeça e Pescoço, Radioterapia, Qualidade de Vida. 
ABSTRACT

Head and neck cancer (HNC) represents a group of malignant tumors and radiotherapy (RT) is a highly used treatment, however it has side effects such as oral mucositis, trismus, xerostomia and / or hyposalivation, among others. Questionnaires were developed to assess the quality of life (QOL) of these patients and the objective of this review was to assess the prevalence of questionnaires for assessing oral and general health of patients undergoing $R T$ for HNC in the literature. Searches in the PubMed ${ }^{\circledR}$ and Lilacs databases were performed with the keywords: "Oral health", "Quality of life", "Head and neck neoplasms", "Radiotherapy", "Oral health", "Quality of life", "Head and neck neoplasms" and "Radiotherapy"; inclusion criteria: articles from QOL questionnaires in patients with HNC treated with RT with/without other antineoplastic treatments; study type: randomized, non-randomized, viability, cross-sectional, linear regression, prospective, longitudinal prospective and randomized prospective; available online; period: 2000-2020, published in Portuguese/English. Total of 18 articles selected using general and global health questionnaires in a patient with HNC. It is concluded that the questionnaire of the European Organization for Research and Treatment of Cancer (specific version for HNC H\& 35 was the most applied followed by its version focused on global health (C30).

Keywords: Head and Neck Neoplasms, Radiotherapy, Quality of Life.

\section{RESUMEN}

Cáncer de cabeza y cuello (CCC) representa grupo de tumores malignos y la radioterapia (RxT) es tratamiento muy utilizado, sin embargo tiene efectos secundarios como mucositis oral, trismo, xerostomia, hiposalivación, entre otros. Se desarrollaron cuestionarios para evaluar la calidad de vida $(C d V)$. El propósito de esta revisión fue evaluar la prevalencia de cuestionarios para evaluar la salud bucal y general de los pacientes sometidos a RxT para CCC en la literatura. Las bases de datos: PubMed® y Lilacs, con las palabras clave: "Salud bucal", "Calidad de vida", "Neoplasias de cabeza y cuello" y "Radioterapia" y "Salud bucal", "Calidad de vida", "Neoplasias de cabeza y cuello", "Radioterapia"; criterios de inclusión: artículos de cuestionarios de calidad de vida en pacientes con CCC tratados con RxT con/sin otros tratamientos antineoplásicos; tipo de estudio: aleatorizado, no aleatorizado, de viabilidad, transversal, de regresión lineal, prospectivo, prospectivo longitudinal y prospectivo aleatorizado; disponible en linea; período: 2000-2020 y publicado en portugués/inglés. Total de 18 artículos seleccionados mediante cuestionarios de salud general y global. Se concluye que el cuestionario de la Organización Europea para la Investigación y el Tratamiento del Cáncer (versión específica para CCC H\&35 fue el más aplicado seguido de su versión para la salud global (C30).

Descriptores: Neoplasias de Cabeza y Cuello, Radioterapia, Calidad de Vida.

\section{INTRODUÇÃO}

O CCP é representado por um grupo de tumores malignos que acometem o trato aerodigestivo superior, é considerado uma doença grave e RxT representa uma das modalidades terapêuticas frequentemente indicada para este tipo de tumor (MENDES H. A. et al., 2017). A RxT em região de cabeça e pescoço resulta em efeitos adversos que acometem a cavidade bucal interferindo em funções como a mastigação, deglutição, fonação, dentre outros, 
reduzindo de forma significativa a $\mathrm{QV}$ desses pacientes (SPECHT, 2002). Desta forma, a Organização Mundial de Saúde iniciou a partir do ano 2000, a elaboração de questionários de QV voltados para a saúde global e a saúde bucal(FISCHER, 2012).

A Qualidade de vida relacionada à saúde global (QVRSG) corresponde a percepção do paciente sobre os efeitos de uma doença e as consequências de seu tratamento, em seus aspectos físicos, psicológicos e sociais (POST, 2014). Atualmente a QVRSG pode ser avaliada por meio de ferramentas genéricas que permitem comparações do impacto da saúde global na QV entre diferentes doenças, ou ferramentas específicas, que por serem mais sensíveis permitem detectar pequenas alterações na QV (MENDONÇA et al., 2019).

A qualidade de vida relacionada a saúde bucal (QVRSB) está relacionada as consequências das complicações bucais geradas pelo tratamento radioterápico. Dentre esses efeitos indesejáveis destacam-se a mucosite oral, xerostomia, hipossalivação, trismo, cárie dentária, disgeusia, disfagia e osteorradionecrose (SPECHT, 2002). Tais complicações resultam no paciente efeitos indesejáveis e prejudiciais nas funções básicas e essenciais da rotina diária como fala, mastigação e deglutição, piorando de forma significativa sua QV(ROSSETTO, 2018).

A QV de pacientes com CCP tem assumido papel de grande interesse na atualidade, sendo observado um número crescente de publicações que utilizam uma ampla variedade de estratégias de avaliação. Entretanto, os artigos encontrados na literatura demonstram que o a maioria dos estudos são referentes a acompanhamentos de curto prazo (WARSHAVSKY et al., 2019). Além disso, tais publicações se baseiam em populações gerais acometidas por $\mathrm{CCP}$, sendo escassos no enfoque ao tratamento radioterápico(SANTOS et al., 2017). O objetivo deste estudo foi identificar a prevalência dos questionários de QV voltados para saúde geral e saúde bucal aplicados em pacientes com CCP tratados por RxT, associado ou não com outros tratamentos antineoplásicos.

\section{MATERIAIS E MÉTODOS}

Este estudo trata-se de uma revisão integrativa que foi realizado respondendo à pergunta: "Quais são os questionários mais prevalentes sobre qualidade de vida geral e/ou relacionada a saúde bucal direcionados a pacientes com CCP tratados por RxT, associado ou não a outros tratamentos antineoplásicos na literatura?". A pesquisa foi realizada nas bases de dados PUBMED/MEDLINE e LILACS, no [PUBMED/MEDLINE] com os descritores, "Oral health", "Quality of life", "Head and neck neoplasms" e "radiotherapy", no [LILACS] os descritores foram "Saúde bucal", "Qualidade de vida", "Neoplasias de cabeça e pescoço" e "Radioterapia", disponíveis online e pelo período de 2000 a 2020.

Os critérios de inclusão foram: 1) artigos que aplicaram questionários de $\mathrm{QV}$ em pacientes com CCP tratados com RxT associado ou não com outros tratamentos antineoplásicos; 2) artigos dos tipos: estudo randomizado, não randomizado, de viabilidade, transversal, regressão linear, prospectivo, prospectivo longitudinal e prospectivo randomizado; 3 ) disponíveis online; 4) por período de 2000 a 2020; 5) publicados em português e inglês.

Os critérios de exclusão foram: 1) artigos que não relacionam o CCP com o tratamento radioterápico em todo grupo de estudo, 2) artigos que não especificam o tipo de questionário de QV aplicado, 3) artigos de revisões e 4) relatos de casos. Para guiar este 
estudo considerou-se: Desenho do estudo, características demográficas, tipo de questionário aplicado, método de avaliação do questionário, resultados e conclusões.

\section{RESULTADOS E DISCUSSÃO}

Foram encontrados 278 artigos, sendo 257 artigos no Pubmed/Medline e 21 artigos no Lilacs (Figura I). Após a aplicação dos critérios de inclusão e exclusão foram selecionados 18 artigos para amostra final. Na figura I observa-se o fluxograma com os resultados encontrados nas bases de dados. Os dados obtidos dos artigos selecionados foram inseridos em tabela em ordem cronológica (Tabela I). Dos 18 artigos incluídos nessa revisão integrativa, o questionário mais citado como critério de avaliação em maior número de artigos foi o questionário da Organização Europeia para Pesquisa e Tratamento do Câncer em sua versão específica para cabeça e pescoço $H \& 35$, com um total de $7(38,9 \%)$ artigos(GRAFF et al., 2007; MCMILLAN et al., 2006; NYQVIST et al., 2016; POW et al., 2006, 2012; RATHOD et al., 2013; YUCE SARI et al., 2016), em sequência com $6(33,3 \%)$ sua versão não específica C30(GRAFF et al., 2007; MCMILLAN et al., 2006; NYQVIST et al., 2016; POW et al., 2006, 2012; RATHOD et al., 2013; YUCE SARI et al., 2016), $5(27,7 \%)$ artigos o questionário UW-QOL(ACKERSTAFF et al., 2002; CHEN et al., 2014; FISHER et al., 2003; OTON-LEITE et al., 2012; PARLIAMENT et al., 2004) e SF-36(FANG et al., 2004; MCMILLAN et al., 2004, 2006; POW et al., 2006, 2012), 4 artigos (22,2\%) questionário OHIP-14 (MCMILLAN et al., 2004; POW et al., 2012; SANTOS et al., 2017; SCHWEYEN et al., 2017), 2 (11,1\%) artigos o questionário XeQoLS(HENSON et al., 2001; PARLIAMENT et al., 2004), 1 artigo (5,5\%) o questionário EQ-5D(RAMAEKERS et al., 2011), 1 artigo $(5,5 \%) \quad$ o questionário FACT-H \&
$\mathrm{N}($ ACKERSTAFF et al., 2002) e 1 (5,5\%) artigo o questionário da Organização Europeia para Pesquisa e Tratamento do Câncer em sua versão específica para câncer de esôfago(YUCE SARI et al., 2016).

Quanto ao tipo de terapia antineoplásica utilizada nos estudos incluídos, 7 pacientes $(38,8 \%)$ relataram receber tratamento radioterápico exclusivo (FISHER et al., 2003; MCMILLAN et al., 2004, 2006; NYQVIST et al., 2016; OTON-LEITE et al., 2012; POW et al., 2006, 2012), 7 pacientes $(38,8 \%)$ receberam quimioterapia concomitante (ACKERSTAFF et al., 2002; CHEN et al., 2014; FISHER et al., 2003; RATHOD et al., 2013; SANTOS et al., 2017; SCHWEYEN et al., 2017; YUCE SARI et al., 2016) e $4(22,2 \%)$ pacientes receberam cirurgia e quimioterapia concomitantes a RxT (FANG et al., 2004; GRAFF et al., 2007; HENSON et al., 2001; RAMAEKERS et al., 2011). Quanto as modalidades de

RxT realizadas 12 artigos $(66,6 \%)$ relataram regime convencional de RxT (FISHER et al., 2003; GRAFF et al., 2007; HENSON et al., 2001; MCMILLAN et al., 2004; NYQVIST et al., 2016; OTON-LEITE et al., 2012; POW et al., 2006, 2012; RAMAEKERS et al., 2011; SANTOS et al., 2017), 9 (50\%) relataram RxT modulada por intensidade (IMRT)(CHEN et al., 2014; FISHER et al., 2003; GRAFF et al., 2007; MCMILLAN et al., 2006; POW et al., 2006, 2012; RATHOD et al., 2013; SCHWEYEN et al., 2017; YUCE SARI et al., 2016), 2 (11,1\%) artigos realizaram RxT de conformidade tridimensional (3DCRT)(RATHOD et al., 2013; SCHWEYEN et al., 2017), $1(5,5 \%)$ realizou regime de fracionamento acelerado (FA)(NYQVIST et al., 2016).

Em relação ao momento do tratamento radioterápico em que o questionário foi aplicado podemos observar que $4(22,2 \%)$ artigos o aplicaram após término o tratamento radioterápico(FANG et al., 
2004; FISHER et al., 2003; GRAFF et al., 2007; RAMAEKERS et al., 2011) e $4(22,2 \%)$ aplicaram antes e após o término(FISHER et al., 2003; HENSON et al., 2001; MCMILLAN et al., 2004; RATHOD et al., 2013), seguido de $3(16,7 \%)$ artigos com aplicação antes, durante e após o término(ACKERSTAFF et al., 2002; NYQVIST et al., 2016; POW et al., 2006), 2 $(11,1 \%)$ com aplicação durante e após término(MCMILLAN et al., 2006; POW et al., 2012), $1(5,5 \%)$ artigo aplicou o questionário durante o tratamento(YUCE SARI et al., 2016), e 1 (5,5\%) antes e durante o tratamento radioterápico(OTON-LEITE et al., 2012).

Resultados para os tipos de questionários encontrados nesta revisão:

A ampla variedade de questionários que avaliam a QV está relacionada as diferentes informações abordadas em cada um deles. Todas essas informações, relacionam as consequências da RxT na QV dos pacientes em sua forma geral (QLQ-C30), específica para o câncer de cabeça e pescoço (H\&N35, OHIP-14, UW-QOL, FACT-H \& N), especifica para suas consequências bucais (XeQoLS), comparada a padrões normais encontrados na população sem a doença e/ou presentes antes da descoberta e/ou finalizado o tratamento (SF-36 e EQ-5D). A diversidade das informações colhidas pelos questionários que avaliam o impacto da saúde bucal na QV além do impacto do CCP na saúde global, nos permita identificar os fatores mais frequentes e prejudiciais, buscando assim medidas preventivas capazes de minimizar essas complicações e reduzir o impacto negativo na $\mathrm{QV}$ dos pacientes submetidos ao tratamento radioterápico. Tal impacto é resultado de consequências bucais geradas pela RxT, como: mucosite oral, xerostomia e/ou hipossalivação, infecções oportunistas, disgeusia, disfagia, cárie de radiação, trismo e/ou fibrose da musculatura irradiada e osteorradionecrose(SANTOS et al., 2017; YUCE SARI et al., 2016).

Os questionários específicos e não específicos mais prevalentes que avaliam as consequências bucais decorrentes da RxT que interferem diretamente nos aspectos físicos, sociais e psicológicos dos pacientes submetidos a esta terapia além de mensurar a interferência na QV geral e/ou relacionada a saúde bucal são: Oral Health Impact Profile (OHIP-14), Questionário central da Organização Européia para Pesquisa e Tratamento do Câncer - EORTC (Módulo básico - QLQ-C30, módulo específico para câncer de cabeça e pescoço - H\&N35 e módulo específico para câncer do esôfago - QLQ-OES18), Questionário de Qualidade de Vida da Universidade de Washington (UW-QOL), Questionary Medical Outcomes Study 36 itens (SF-36), Questionário Euroqol-5D (EQ-5D), Questionário de Avaliação Funcional de Terapia do Câncer Cabeça e Pescoço (FACT-H \& N), Questionário de qualidade de vida relacionado a xerostomia (XeQoLS); totalizando 9 tipos de questionários diferentes.

Dentre os questionários de QV específicos para CCP, o questionário OHIP-14 analisa o impacto da saúde bucal na QV através da avaliação de 7 dimensões: limitação funcional, desconforto psicológico, incapacidade social, deficiência, dor física, incapacidade física e incapacidade psicológica(MCMILLAN et al., 2004; POW et al., 2012; SANTOS et al., 2017; SCHWEYEN et al., 2017). O questionário UW-QOL também avalia o impacto da saúde bucal na QV, entretanto de forma distinta, onde são considerados 12 domínios, como: dor, aparência, atividade, recreação, deglutição, mastigação, fala, função do ombro, sabor, saliva, humor e ansiedade (CHEN et al., 2014; FISHER et al., 
2003; OTON-LEITE et al., 2012; PARLIAMENT et al., 2004). Outros aspectos possíveis de serem avaliados são estados de saúde geral, funcional e sintomático, com associação de perguntas relacionadas a fala, mastigação e deglutição, presente no questionário EORTC em sua versão específica para CCP (H\&N35) e esôfago (OES18) (GRAFF et al., 2007; MCMILLAN et al., 2004; NYQVIST et al., 2016; POW et al., 2006, 2012; RATHOD et al., 2013; YUCE SARI et al., 2016). Por fim, o questionário FACT-H \& N permite a avaliação através de seus 28 itens e 6 subescalas do bem-estar físico, bem-estar social e familiar, relação com o médico, bem-estar emocional, bem-estar funcional e sintomas específicos da cabeça e pescoço, aonde maiores scores nas várias subescalas representam uma melhor QV(ACKERSTAFF et al., 2002).

Com relação aos questionários que avaliam a QV geral do paciente, o questionário SF- 36 apresenta 36 questões que são divididas em 8 subescalas que avaliam o funcionamento físico, funcionamento social, limitação do papel-físico, limitação do papelemocional, saúde mental, vitalidade, dor e percepção geral de saúde (FANG et al., 2004; MCMILLAN et al., 2004, 2006; POW et al., 2006). Outros aspectos possíveis de serem avaliados são estados de saúde geral, funcional e sintomático, presente nos questionários EORTC, em sua versão geral (QLQ-C30) e específicas para câncer de esôfago (QLQOES18)(GRAFF et al., 2007; POW et al., 2006, 2012). O questionário EQ-5D, por sua vez, permite avaliar 5 dimensões: mobilidade, autocuidado, atividades habituais, dor/desconforto e ansiedade/depressão (RAMAEKERS et al., 2011).

Henson et al., identificaram impactos variáveis na QV quando compararam diferentes modalidades de RxT (convencional X acelerado), onde observou-se que o acelerado gera um pior efeito adverso na QVRS na fase aguda do que o convencional(HENSON et al., 2001). A magnitude do comprometimento da QV é menor e a recuperação mais rápida com a modalidade de RxT IMRT em comparação a 3D-CR, considerando os desfechos da doença, assim como seus benefícios nas áreas de desconforto bucal e disfunção salivar (GRAFF et al., 2007; HENSON et al., 2001). A modalidade IMRT para doença em estágio inicial apresenta uma grande vantagem por preservar aspectos-chave da QV (MCMILLAN et al., 2006). A avaliação do tempo de permanência das sequelas decorrentes do tratamento radioterápico, mostrou que o impacto da saúde bucal na QV foi menor após o término do tratamento quando comparado ao momento do diagnóstico do câncer e durante o tratamento (CHEN et al., 2014). Todavia, as sequelas, embora em menor quantidade, permaneceram mesmo após a finalização do tratamento, apresentando naqueles com idade mais avançada, menor renda familiar anual, com estágio de câncer mais avançado, uma interferência física significativamente pior, e aqueles com menor renda familiar anual, desemprego e CCP em estágio mais avançado uma interferência mental significativamente pior (FANG et al., 2004). Desse modo, não se pode negar que sobreviventes de câncer em cavidade oral vivem com pior QVRSB em comparação com a população geral e que fatores socioeconômicos e estágio do câncer são importantes correlacionados com a QV(FANG et al., 2004). Pacientes com tumores localizados na cavidade oral apresentam impacto na QVRSG significativamente maior do que pacientes com outros locais de tumor(SCHWEYEN et al., 2017). 
Figura 1. Fluxograma com os resultados encontrados nas bases de dados.

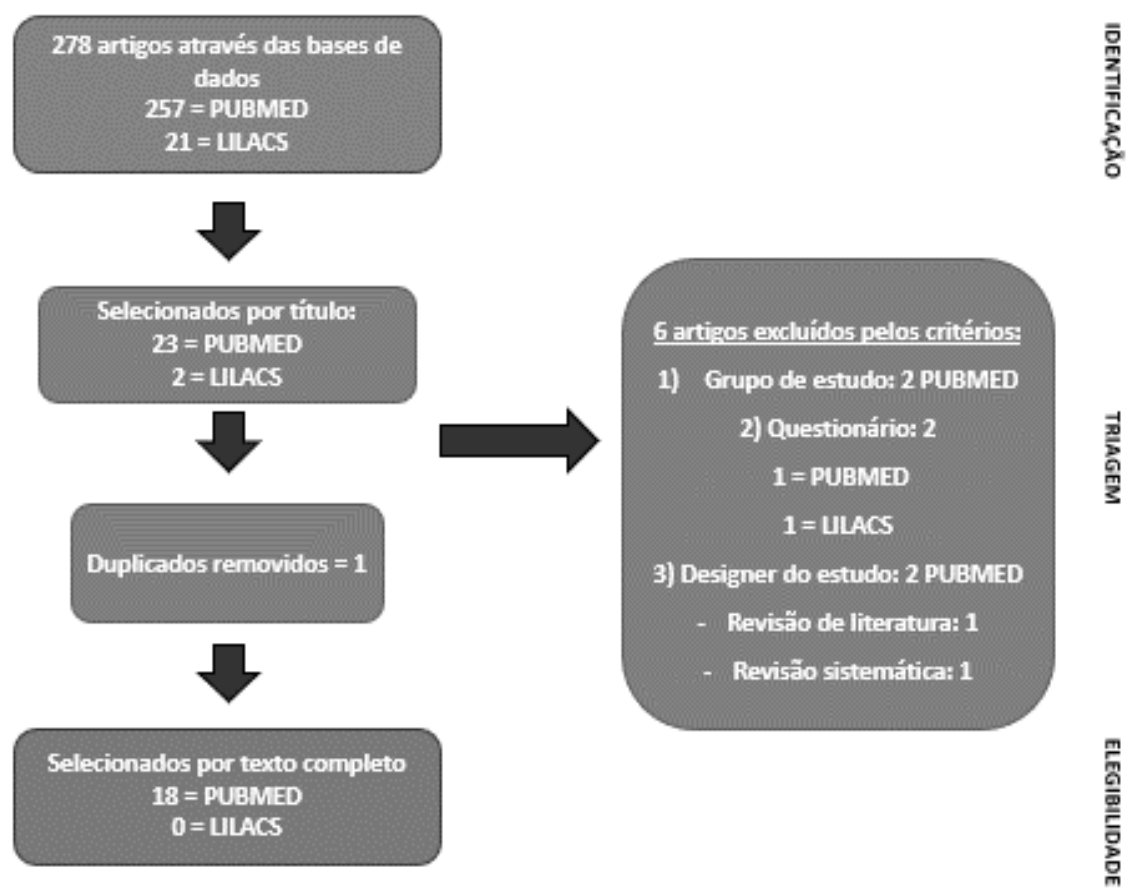

Tabela 1. Artigos encontrados nas bases de dados Pubmed/Medline e Lilacs.

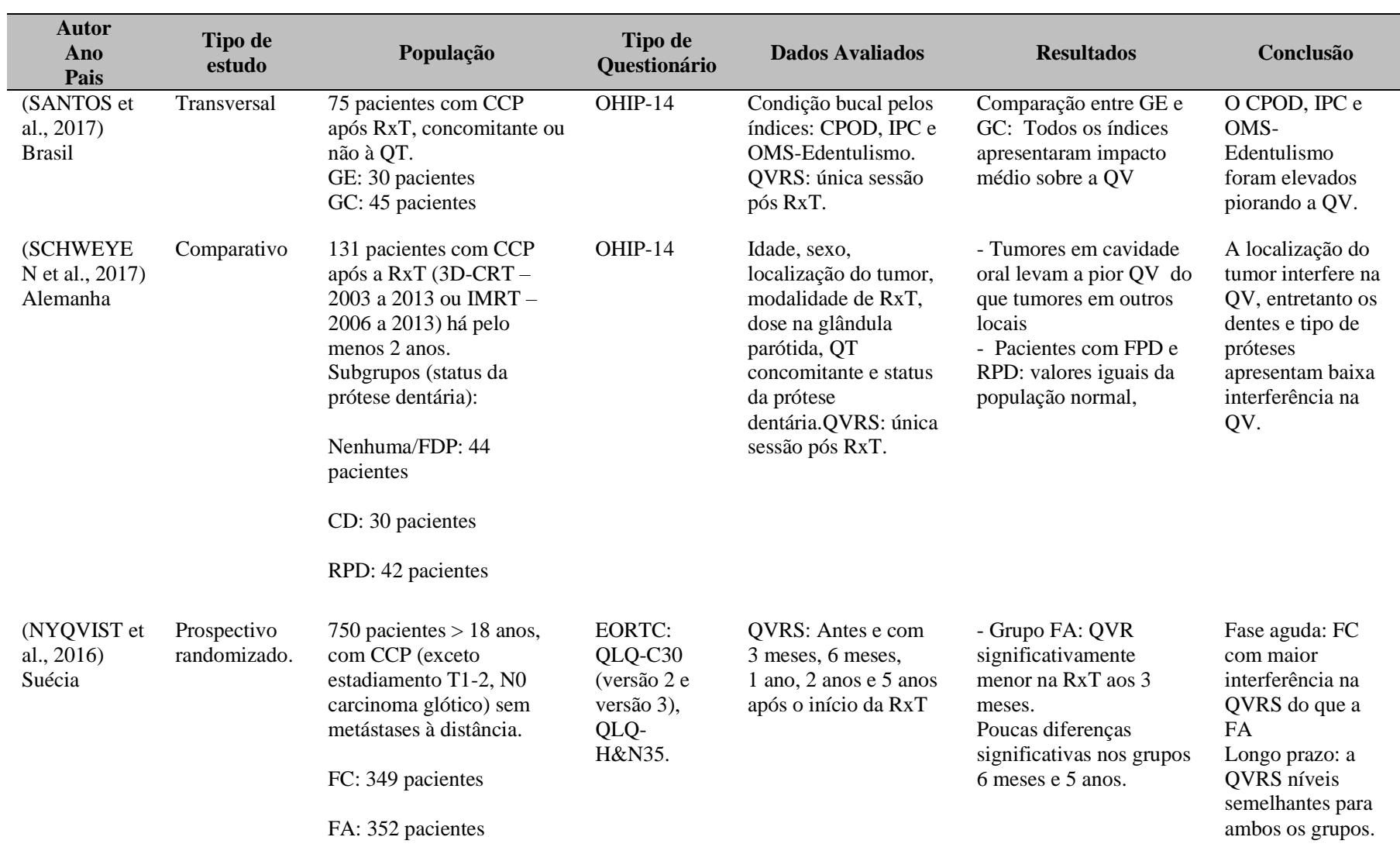




\begin{tabular}{|c|c|c|c|}
\hline $\begin{array}{l}\text { (YUCE SARI } \\
\text { et al., 2016) } \\
\text { Turquia }\end{array}$ & $\begin{array}{l}\text { Pesquisa } \\
\text { clínica (não } \\
\text { randomizado) }\end{array}$ & $\begin{array}{l}29 \text { pacientes com CCP } \\
\text { submetidos a RxT IMRT. } \\
\text { Teste de GAE: } 15\end{array}$ & $\begin{array}{l}\text { EORTC: } \\
\text { QLQ-C30, } \\
\text { QLQ-H\&N35 } \\
\text { e QLQ- } \\
\text { OES18 }\end{array}$ \\
\hline
\end{tabular}

Sem o teste: 14

$\begin{array}{lll}\text { (CHEN et al., } & \text { Transversal } & 50 \text { pacientes com CCP } \\ \text { 2014) } & & \text { avançado submetidos a } \\ \text { Estados } & \text { IMRT bilateral sem } \\ \text { recorrência, com mínimo } \\ \text { de } 5 \text { anos de seguimento. }\end{array}$

$\begin{array}{lll}\text { (RATHOD et } & \text { Randomizado } & \text { 60 pacientes com CCP } \\ \text { al., 2013) } & \cdot & \begin{array}{l}\text { submetidos a RxT 3D-CRT } \\ \text { ou IMRT }\end{array} \\ \text { India } & & \end{array}$

3D-CRT: 28 pacientes

IMRT: 32 pacientes

\begin{tabular}{|c|c|c|}
\hline $\begin{array}{l}\text { (OTON- } \\
\text { LEITE et al., } \\
2012) \\
\text { Brasil }\end{array}$ & Randomizado & $\begin{array}{l}60 \text { pacientes com CCP (> } \\
18 \text { anos) pós RxT } \\
\text { envolvendo principais } \\
\text { glândulas salivares. } \\
\text { Grupo Laserterapia: } 30 \\
\text { pacientes } \\
\text { Grupo Placebo: } 30 \\
\text { pacientes }\end{array}$ \\
\hline $\begin{array}{l}\text { (POW et al., } \\
\text { 2012) } \\
\text { China }\end{array}$ & $\begin{array}{l}\text { Prospectivo } \\
\text { longitudinal. }\end{array}$ & $\begin{array}{l}57 \text { pacientes com CCP } \\
\text { (estágio inicial) submetidos } \\
\text { a IMRT }\end{array}$ \\
\hline
\end{tabular}

$\begin{array}{lll}\text { (RAMAEKE } & \text { Transversal } & \text { 396 pacientes com CCP } \\ \text { RS et al., } & & \begin{array}{l}\text { tratados com RxT após 6 } \\ \text { meses, sem recorrência da } \\ \text { 2011) }\end{array} \\ \text { Holanda } & & \begin{array}{l}\text { doença. }\end{array}\end{array}$

$\begin{array}{lll}\text { (GRAFF et } & \text { Transversal } & \text { 134 pacientes com CCP } \\ \text { al., 2007) } & & \text { tratados com RxT após } 1 \\ \text { França } & \text { ano. } \\ & \text { IMRT: } 67 \text { pacientes } \\ & \text { RxT convencional: } 67 \\ & \text { pacientes }\end{array}$

UW-QOL.

EORTC:

QLQ-C30 e QLQ-H\&N35

UW-QOL

SF- 36,

EORTC:

QLQ-C30 e QLQ-

H\&N35, e OHIP-14

EQ-5D. QLQ-

$\mathrm{H} \& \mathrm{~N} 35$.
QVRS: $1^{\circ}, 15^{\circ} \mathrm{e}$

última sessão da RxT

Escore global (estado de saúde, funcional e escala de sintomas): $1^{\circ}$ dia: semelhante nos dois $15^{\circ} \mathrm{e}$ última sessão: os escores das funções sociais, dor, apetite, boca seca, saliva, problemas com paladar, alimentação, social e de deglutição foram piores no grupo controle.

QVRS pós RxT: 2 a 4 semanas, a cada 2 a 3 meses no $1^{\circ}$, a cada 4 a 6 meses no $2^{\circ} \mathrm{e} 3^{\circ}$ anos e após 1 avaliação/ano

QVRS: Pré-tratamento e $3,6,12,18$ e 24 meses pós RxT.

QVRS: Antes e após $15^{\circ}$ e $30^{\circ}$ sessão

Diminuição QV em ambos os grupos, entretanto menor interferência no grupo de laserterapia.

Influência da
toxicidade tardia
(xerostomia e disfagia)
com RxT isolada ou
combinada com
cirurgia e/ou
quimioterapia. QVRS:
Única sessão pós RxT.

Medição do fluxo
salivar da parótida e
do fluxo total. QVRS:
Início, 2, $6,12,18$ e 24
meses após a IMRT.

$1^{\circ}$ ano: Recuperação total do fluxo salivar da parótida e parcial do fluxo total, com diminuição da $\mathrm{QV}$ após a IMRT, seguida de recuperação gradual. $2^{\circ}$ ano: Persistente de fluxo total de forma parcial

\begin{tabular}{|c|c|}
\hline $\begin{array}{l}\text { A disfagia tem um } \\
\text { impacto negativo maior } \\
\text { na QVRS quando } \\
\text { comparada a xerostomia; } \\
\text { embora ambas } \\
\text { apresentem um impacto } \\
\text { negativo na QVRS }\end{array}$ & $\begin{array}{l}\text { A redução de } \\
\text { toxicidade da RxT } \\
\text { é maior nos } \\
\text { pacientes com } \\
\text { risco de } \\
\text { xerostomia e/ou } \\
\text { disfagia. }\end{array}$ \\
\hline $\begin{array}{l}\text { Menor impacto negativo } \\
\text { no grupo IMRT, } \\
\text { (disfunção salivar). } \\
\text { Sintomas mais graves e } \\
\text { mais frequentes com } \\
\text { RxT convencional } \\
\text { (alterações salivares e } \\
\text { desconforto bucal). }\end{array}$ & $\begin{array}{l}\text { IMRT resulta } \\
\text { menor impacto } \\
\text { negativo na QV } \\
\text { relacionada a } \\
\text { disfunção salivar e } \\
\text { desconforto bucal. }\end{array}$ \\
\hline
\end{tabular}

- Impacto negativo na QVRS pela RxT no entanto, o uso do GAES pode mediar esse efeito negativo.

A melhora da QV após RxT comprova a preservação de estruturas adjacentes quando tratados com IMRT.

Após a RxT há uma diminuição gradual na $\mathrm{QV}$, que melhorou com o tempo nas duas modalidades de RxT. A QV é menos comprometida e com recuperação mais rápida na IMRT.

A laserterapia reduz o impacto negativo da RxT na QV de pacientes com CCP.

IMRT em Carcinoma Nasofaríngeo (estágio inicial) preserva parcialmente a função salivar e QVRS

EORTC: $\quad$ Comparação IMRT e QLQ-C30 RxT convencional. (versão 3,0) e QVRS: Única sessão pós RxT. 


\begin{tabular}{lllll}
\hline MCMILLA & Prospectivo & 32 pacientes com CCP & SF-36 e & Medição do fluxo \\
N et al., 2006) & & (Estadiamento T1-2, N0-1, & EORTC: & salivar. QVRS: Início, \\
China & M0 NPC) tratados com & QLQ- & 2, 6 e 12 meses após a \\
& IMRT. & H\&N35. & IMRT.
\end{tabular}

2 meses: quadros de
xerostomia, fadiga,
vitalidade foram citados.
2,3 e 12 meses:
melhora na QV.
$1^{\circ}$ ano: recuperação de
$25 \%$ do fluxo salivar na
maioria dos casos.

(POW et al.,

2006)

China

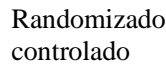

tratado com IMRT e RxT

convencional.

IMRT: 25 pacientes

RxT convencional: 26

pacientes

(FANG et al.

2004)

China

Comparativo.

66 pacientes com $\mathrm{CCP}$

após cirurgia e RxT

adjuvante com

acompanhamento $>2$ anos

SF-36 (versão Avaliação

de Taiwan)

sociodemográfica e

médicas

correlacionadas com o

resumo de PCS e de

MCS das populações

taiwanesas e norte-

americanas. QVRS

Única sessão no

último

acompanhamento pós RxT.

2 meses: relato de

alteração salivar e

melhora na QVRS após

os dois tratamentos.

12 meses: IMRT com

melhora na QV com

xerostomia menos citada

e melhor condição bucal

do que na na RxT

convenciona.

Idade avançada, baixa

renda, estágio de câncer

mais avançado e

reconstrução de retalho,

tiveram escores piores

do PCS. Por outro lado,

aqueles com baixa renda,

desemprego e estágio

mais avançado do câncer

relataram MCS

significativamente pior.

Medida de boca seca e

QVRS: SCCP -

completado 1 ano da

RxT. NCCP - Antes

da RxT e tratamento

odontológico prévio.

NCCP: 40 pacientes

GC: 31 pacientes

23 pacientes com $\mathrm{CCP}$

Estudo de viabilidade de

Fase I-II

NT et al.,

2004)

Canada
UW-QOL e XeQoLS.

Medição das taxas de
fluxo salivar não
estimuladas e
estimuladas. QVRS no
primeiro ano após
RxT: 1,3 e 12 meses,
para 22,22 e 18
pacientes,
respectivamente.

O fluxo salivar estimulado e não estimulado foram preservados. A proporção de pacientes com xerostomia leve ou ausente permaneceram constantes durante a pesquisa.

Distribuição por raça, sexo, uso de tabaco, local do tumor, estágio $\mathrm{T}$ e função salivar. Grupo placebo: maior dor bucal e dificuldade mastigatória

Grupo pilocarpina:

maior dificuldade de deglutição,

hipossalivação e gosto prejudicado.

Mucosite oral igual aos 3 meses em ambos os grupos, com necessidade de aumento nutricional
IMRT mostrou

redução nos

sintomas da

xerostomia,

resultando em

impacto negativo

menor na QV.

É notado

benefício

significativo da

IMRT resulta em

maior preservação

da glândula

parótida e melhor

desfecho de QV.

Observou-se pior

QVRS da

população norte

americana em

comparação com a

de

Taiwan. Fatores

socioeconômicos

e estágio do

câncer foram

fatores

importantes

correlacionados

com a QVRS.

A importância dos

critérios de

avaliação da

QVRS leva a

compreensão dos

diferentes

impactos

psicológicos,

sociais e físicos

nas diversas fases

de tratamento.

A QVRS foi

preservada nos 12

meses após

IMRT. É

necessário um

acompanhamento

a longo prazo para

avaliar em que

medida a QVRS é

mantida de forma

favorável.

A prevenção da hipossalivação

não afetou a

avaliação da

função salivar ou

da QV dos

pacientes, devido

ao maior impacto

da mucosite na

QV após a RxT.

Grupo Pilocarpina: 121

pacientes

Grupo Placebo: 125

pacientes

*exclusão de 33 pacientes

que estavam em

desconformidades. 


\begin{tabular}{|c|c|c|c|c|c|c|}
\hline $\begin{array}{l}\text { (ACKERSTA } \\
\text { FF et al., } \\
\text { 2002) } \\
\text { Holanda }\end{array}$ & $\begin{array}{l}\text { Viabilidade } \\
\text { não } \\
\text { randomizado. }\end{array}$ & $\begin{array}{l}50 \text { pacientes com CCP } \\
\text { inscritos no RADPLAT. } \\
\text { Grupo falha: } 24 \text { pacientes } \\
\text { Grupo QV } 1 \text { ano: } 26 \\
\text { pacientes }\end{array}$ & $\begin{array}{l}\text { FACT-H\&N } \\
\text { e UW-QOL. }\end{array}$ & $\begin{array}{l}\text { QVRS: antes e, } 3,6 \text { e } \\
12 \text { meses após o início } \\
\text { da RxT. }\end{array}$ & $\begin{array}{l}21 \text { pacientes retornaram } \\
\text { a uma dieta oral, } \\
\text { enquanto } 5 \text { pacientes } \\
\text { ainda precisavam de } \\
\text { alimentação via sonda } \\
\text { nasoenteral. } 10 \\
\text { pacientes retornaram ao } \\
\text { trabalho. A xerostomia } \\
\text { foi relatada por } 17 \\
\text { pacientes. }\end{array}$ & $\begin{array}{l}\text { Resultados } \\
\text { ressaltam a } \\
\text { viabilidade do } \\
\text { protocolo } \\
\text { RADPLAT. }\end{array}$ \\
\hline $\begin{array}{l}\text { (HENSON et } \\
\text { al., 2001) } \\
\text { Estados } \\
\text { Unidos }\end{array}$ & - & $\begin{array}{l}20 \text { pacientes com CCP } \\
\text { tratados por RxT bilateral } \\
\text { com preservação de } \\
\text { parótida cervical em até } 1 \\
\text { ano. }\end{array}$ & XeQoLS & $\begin{array}{l}\text { QVRS: antes e } 1,3,6 \\
\text { e } 12 \text { meses finalizado } \\
\text { a RxT. Fluxo salivar } \\
\text { avaliado com técnicas } \\
\text { de } 3 \text {-DTP e se possui } \\
\text { resultados melhores no } \\
\text { XeQoLS. }\end{array}$ & $\begin{array}{l}\text { Diminuição do fluxo } \\
\text { salivar e aumento da } \\
\text { função de UPFR e SPFR } \\
\text { poupada após RxT, além } \\
\text { de piora no XeQoLS. } \\
\text { Melhora na QV após } 1 \\
\text { mês e } 1 \text { ano concluído a } \\
\text { RxT. }\end{array}$ & $\begin{array}{l}\text { Resultados do } \\
\text { XeQoLS: técnicas } \\
\text { de RxT que } \\
\text { preservam a } \\
\text { parótida são } \\
\text { benéficas. }\end{array}$ \\
\hline
\end{tabular}

\section{CONCLUSÃO}

Os questionários de avaliação da QV voltados para saúde global e bucal são de suma importância para abranger o paciente de forma ampla e completa, favorecendo assim a prevenção das complicações decorrentes da RxT bem como o restabelecimento da QV sob os aspectos físico, psicológicos e sociais. $\mathrm{O}$ questionário da Organização Europeia para Pesquisa e Tratamento do Câncer em sua versão específica para cabeça e pescoço H\&35 foi o mais prevalente na literatura seguido de sua versão voltada para avaliação a saúde global (C30).

\section{AGRADECIMENTO}

O presente trabalho foi realizado com apoio da Coordenação de Aperfeiçoamento de Pessoal de Nível Superior - Brasil (CAPES) - Código de Financiamento 001.

Prof. Dr. Paulo Sérgio da Santos recebeu bolsa do $\mathrm{CNPq}$ processo $\mathrm{n}^{\mathrm{o}}$. 309525/2018-7 durante a realização do estudo.

Todos os autores declararam não haver qualquer potencial conflito de interesses referente a este artigo.

\section{REFERÊNCIAS}

ACKERSTAFF, A. H. et al. Quality-of-life assessment after supradose selective intra-arterial cisplatin and concomitant radiation (RADPLAT) for inoperable stage IV head and neck squamous cell carcinoma. Archives of Otolaryngology - Head and
Neck Surgery, v. 128, n. 10, p. 1185-1190, 2002.

ROSSETTO, MAAM. Qualidade De Vida Em Pacientes Com Câncer De Cabeça E Pescoço. Piracicaba, SP. Dissertação de Mestrado. Faculdade De Odontologia De Piracicaba -Universidade Estadual De Campinas (UNICAMP); 2018.

CHEN, A. M. et al. Quality of life among long-term survivors of head and neck cancer treated by intensitymodulated radiotherapy. JAMA Otolaryngology Head and Neck Surgery, v. 140, n. 2, p. 129-133, 2014.

FANG, F. M. et al. Health-related quality of life outcome for oral cancer survivors after surgery and postoperative radiotherapy. Japanese Journal of Clinical Oncology, v. 34, n. 11, p. 641-646, 2004.

FISCHER, F. M. Relevância dos fatores psicossociais do trabalho na saúde do trabalhador. Revista de Saúde Pública, v. 46, n. 3, p. 401-406, jun. 2012.

FISHER, J. et al. Phase III quality-of-life study results: Impact on patients' quality of life to reducing xerostomia after radiotherapy for head-and-neck cancer - RTOG 97-09. International Journal of Radiation Oncology Biology Physics, v. 56, n. 3, p. 832-836, 2003.

GRAFF, P. et al. Impact of Intensity-Modulated Radiotherapy on Health-Related Quality of Life for Head and Neck Cancer Patients: Matched-Pair Comparison with Conventional Radiotherapy. International Journal of Radiation Oncology Biology Physics, v. 67, n. 5, p. 1309-1317, 2007.

HENSON, B. S. et al. Preserved salivary output and xerostomia-related quality of life in head and neck cancer patients receiving parotid-sparing radiotherapy. Oral Oncology, v. 37, n. 1, p. 84-93, 2001.

MCMILLAN, A. S. et al. Oral health-related quality of life in southern Chinese following radiotherapy for nasopharyngeal carcinoma. Journal of Oral 
Rehabilitation, v. 31, n. 6, p. 600-608, 2004.

MCMILLAN, A. S. et al. Preservation of quality of life after intensity-modulated radiotherapy for early-stage nasopharyngeal carcinoma: Results of a prospective longitudinal study. Head \& Neck, v. 28, n. 8, p. 712 722, ago. 2006.

MENDES H. A.; RODRIGUES-NETO, J. F.; LEITE, MAISA TAVARES DE SOUZA; SAMPAIO, C. A., P. H. C.; B. Significado Das Sequelas Faciais Estéticas Para Indivíduos Submetidos À Cirurgia Para Tratamento De Câncer De Cabeça E Pescoço. Unimontes Científica, v. 19, p. 142-152, 2017.

MENDONÇA, R. B. et al. Evaluation of the measurement properties of the Brazilian version of two quality-of-life questionnaires in food allergy - for children and their parents. Jornal de Pediatria, n. xx, 2019.

NYQVIST, J. et al. Differences in health related quality of life in the randomised ARTSCAN study; Accelerated vs. conventional radiotherapy for head and neck cancer. A five year follow up. Radiotherapy and Oncology, v. 118, n. 2, p. 335-341, 2016.

OTON-LEITE, A. F. et al. Effect of intraoral low-level laser therapy on quality of life of patients with head and neck cancer undergoing radiotherapy. Head \& Neck, v. 34, n. 3, p. 398-404, mar. 2012.

PARLIAMENT, M. B. et al. Preservation of oral health-related quality of life and salivary flow rates after inverse-planned intensity- modulated radiotherapy (IMRT) for head-and-neck cancer. International Journal of Radiation Oncology Biology Physics, v. 58, n. 3, p. 663-673, 2004.

POST, M. W. M. Definitions of quality of life: What has happened and how to move on. Topics in Spinal Cord Injury Rehabilitation, v. 20, n. 3, p. 167-180, 2014.

POW, E. H. N. et al. Xerostomia and quality of life after intensity-modulated radiotherapy vs. conventional radiotherapy for early-stage nasopharyngeal carcinoma: Initial report on a randomized controlled clinical trial. International
Journal of Radiation Oncology Biology Physics, v. 66, n. 4, p. 981-991, 2006.

POW, E. H. N. et al. Can intensity-modulated radiotherapy preserve oral health-related quality of life of nasopharyngeal carcinoma patients? International Journal of Radiation Oncology Biology Physics, v. 83, n. 2, p. e213-e221, 2012.

RAMAEKERS, B. L. T. et al. The impact of late treatment-toxicity on generic health-related quality of life in head and neck cancer patients after radiotherapy. Oral Oncology, v. 47, n. 8, p. 768-774, 2011.

RATHOD, S. et al. Quality-of-life (QOL) outcomes in patients with head and neck squamous cell carcinoma (HNSCC) treated with intensity-modulated radiation therapy (IMRT) compared to three-dimensional conformal radiotherapy (3D-CRT): Evidence from a prospective randomized s. Oral Oncology, v. 49, n. 6, p. 634-642, 2013.

SANTOS, P. S. et al. The impact of oral health on quality of life in individuals with head and neck cancer after radiotherapy: the importance of dentistry in psychosocial issues. Acta odontologica latinoamericana : AOL, v. 30, n. 2, p. 62-67, 2017.

SCHWEYEN, R. et al. The impact of oral rehabilitation on oral health-related quality of life in patients receiving radiotherapy for the treatment of head and neck cancer. Clinical Oral Investigations, v. 21, n. 4, p. 1123-1130, 2017.

SPECHT, L. Oral complications in the head and neck radiation patient: Introduction and scope of the problem. Supportive Care in Cancer, v. 10, n. 1, p. 36-39, 2002.

WARSHAVSKY, A. et al. Long-term health-related quality of life after mandibular resection and reconstruction. European Archives of Oto-RhinoLaryngology, v. 276, n. 5, p. 1501-1508, 2019.

YUCE SARI, S. et al. The effect of glutamine and arginine-enriched nutritional support on quality of life in head and neck cancer patients treated with IMRT. Clinical Nutrition ESPEN, v. 16, p. 30-35, 2016. 